\title{
Unruptured Vertebral Artery Dissecting Aneurysms: Approach Strategy by Retrospective Analysis
}

\author{
Arash Dooghaie Moghadam, Ali Keipourfard², Yasaman Arjmand ${ }^{1, *}$ \\ ${ }^{1}$ Department of Radiology, Shohada Tajrish Hospital, Shahid Beheshti University of Medical Sciences, Tehran, Iran \\ ${ }^{2}$ Bone Joint and Related Tissue Research Center, Akhtar Hospital, Shahid Beheshti University of Medical Sciences, Tehran, Iran \\ *Corresponding author: yasi.arj@gmail.com
}

\begin{abstract}
Objectives: The natural course of un-ruptured vertebral artery dissecting aneurysms (VADAs) is not completely clear. We aim to retrospectively develop a strategy for treating un-ruptured VADAs based on long-term follow-up. Methods: We retrospectively studied 35 patients with un-ruptured VADAs. The initial symptom of 20 patients was headache, followed by ischemic symptoms and mass effect in 11 and 4 patients respectively. All of the patients underwent Magnetic Resonance Imaging (MRI) and Magnetic Resonance Angiography (MRA) at the time of admission and 2 weeks and 1, 4, 6, 12, and 24 months after initial presentation. Asymptomatic patients with enlarging dissection site defined on MRI and MRA, received further treatment and work up. Results: Two patients received emergency intervention due to symptom exacerbation and unstable status. The other 33 patients underwent conservative management. Lesion enlargement was observed in 2 cases during imaging follow up. In follow up period, additional interventions including dissection trap by surgery and coil embolization were conducted in 1 and 3 patients respectively. Other 31 patients remain symptom free and were managed conservatively. Dissection site remained unchanged in majority of patients (68.57\%), improved in $28.57 \%$ and disappeared in $2.85 \%$ of the patients. Ten patients with recurrent ischemic attacks underwent anti-platelet therapy, without any bleeding complaint or permanent neurological deficits. Conclusion: The nature of an un-ruptured VADA is not highly aggressive. However, enlarged dissection site without new manifestations, occlusion is recommended. Also, anti-platelet therapy is suggested in patients with recurrent ischemic attacks.
\end{abstract}

Keywords: vertebral artery dissecting aneurysm, magnetic resonance imaging, conservative treatment, recurrent ischemic attacks, anti-platelet therapy

Cite This Article: Arash Dooghaie Moghadam, Ali Keipourfard, and Yasaman Arjmand, "Unruptured Vertebral Artery Dissecting Aneurysms: Approach Strategy by Retrospective Analysis.” American Journal of Medical Case Reports, vol. 5, no. 8 (2017): 202-204. doi: 10.12691/ajmcr-5-8-2.

\section{Introduction}

Dissection of an un-ruptured intracranial vertebral artery aneurysm (VAA) is one of the most identifiable causes of stroke in young adults and will manifest either as severe headache in occipital lobe or focal neurological deficits (transient or permanent) caused by vertebro-basilar artery ischemia. [1,2,3] These lesions tend to occur equally in men and women in their 40s and also are more common in patients with connective tissue disorders. [4] Subarachnoid hemorrhage (SAH) is the primary manifestation in patients with ruptured VAAs. The natural course of VAA strongly depends on primary manifestation. Due to high mortality rate of acute phase re-bleeding, open surgery or endovascular procedures are commonly performed in patients presenting with SAH or unstable status. [5] Satisfactory prognosis and non-aggressive nature of un-ruptured VAAs, advocates conservative management of these lesions. [6]

Since there is no unified and acceptable follow-up and treatment have not been defined, we plan to investigate the natural history and of un-ruptured vertebral artery dissecting aneurysms (VADAs).

\section{Methods}

Between October 2011 and March 2016, 20 male and 15 female patients $(\mathrm{N}=35)$ with un-ruptured VADAs with signed informed patient consent form were admitted to Shohada Tajrish Hospital. Stable patients with isolated headache were treated conservatively, while patients with progressive ischemic and mass effect symptoms received anti-platelet and steroid therapy respectively. Angiography was performed in patients who fail to improve despite mentioned appropriate treatments. Patient's systolic blood pressure was maintained at $140 \mathrm{mmHg}$ or bellow. All of the patients were studied by MRI and MRA, by focusing on series of diagnostic criteria, such as stenotic segments as string sign, occluded segments, pseudo-aneurysm and irregularity of lumen. MRI and MRA were performed at the end of the second week, followed by $1^{\text {st }}, 4^{\text {th }}, 6^{\text {th }}, 12^{\text {th }}$ and $24^{\text {th }}$ months later. Asymptomatic patients with enlarging dissection site on MRI and MRA and patients with progressive focal neurological symptoms despite anti-platelet therapy underwent angiography, by a 6French guiding catheter and a non-detachable balloon as a 
test for occlusion in affected VADA. Arterial occlusion was made by contrast media inflation in target balloon. During the procedure, vital signs were monitored every 15 to 20 minutes and serial neurosurgical examinations were performed. Also, the contra-lateral VA flow and possible retrograde aneurysm filling were recorded. Trapping with Guglielmi detachable coils (Boston Scientifics, Natick, MA, USA) using double micro-catheter technique was performed in VADAs which did not involve posterior inferior cerebellar artery (PICA). Patients with PICA involvement underwent direct aneurysm trap by open surgery and PICA reconstruction via occipital arteryPICA anastomosis. This study was conducted under the principles of the Helsinki Declaration and approved by the Ethics Committee of Shohada Tajrish hospital.

\section{Results}

The mean age of the patients was 55.1 years (28-80 years old). The initial symptom of 20 patients was headache, followed by ischemic symptoms and mass effect in 11 and 4 patients respectively. In the first 2 weeks of symptom onset, 2 patients experienced initial ischemic symptom exacerbation despite appropriate antiplatelet therapy. Proximal occlusion of VA by Guglielmi detachable coil was performed in one patient, while the other one underwent direct dissection site trap by open surgery due to PICA involvement. Also, an occipital artery to PICA anastomosis was performed in later patient. Conservative management was performed for other 33 patients by blood pressure control (systolic blood pressure less than $140 \mathrm{mmHg}$ ). In addition, all of the patients underwent MRI and MRA study at the end of the second week, followed by $1^{\text {st }}, 4^{\text {th }}, 6^{\text {th }}, 12^{\text {th }}$ and $24^{\text {th }}$ months. At the end of the second week, 1 patient experienced dysphagia due to mass effect that underwent coil embolization. Also, at the end of the $4^{\text {th }}$ month, another patient showed asymptomatic dissection site enlargement on MRA who also underwent same procedure as previous patient. At later follow-ups on $6^{\text {th }}, 12$ th and $24^{\text {th }}$ month, no patient experienced additional symptoms. Finally 2 patients were treated in acute stage before first follow-up visit by direct surgery and interventional treatment, and another 2 patients were managed by interventional procedure during follow-up period. Of all 3 patients who received interventional treatment, 2 patients were managed by proximal occlusion while the other one received coil trapping. Of all patients who received either interventional or surgical management $(\mathrm{N}=4)$, no one encountered excessive bleeding, new neurological deficits or aneurismal rupture. Rest of the patients $(\mathrm{N}=31)$ showed no new neurological deficits or mass effect symptoms in whole follow-up period. Twenty four (68.57\%) patients showed no further changes on MRI and MRA studies. The morphology of the dissection site was aneurysmal dilation $(\mathrm{N}=5)$ and pearl-and-string sign $(\mathrm{N}=19)$. Interestingly, isolated string sign was not observed in this group of patients. Of note, MRI and MRA studies revealed signs of healing in dissection site in follow-up period in 10 patients (28.57\%). Mentioned dissections were 2 aneurysmal dilation, 7 pearl-and-string sign and 1 isolate string sign on imaging evaluations. One patient (2.85\%) experienced dissection site disappearance at the end of the $4^{\text {th }}$ month, documented by MRI and MRA, without developing any new neurological or mass effect symptoms or signs of recanalization of the site. Anti-platelet therapy was prescribed for 10 patients with recurrent ischemic attacks, who presented with ischemic attacks and headache. Pearland-string sign and aneurismal dilation was observed equally in these patients on MRI and MRA studies. No new or permanent focal neurological deficits and ischemic attacks were recorded for patients receiving anti-platelet therapy which was continued for at least 2 years.

\section{Discussion}

Although most of the un-ruptured VADAs do not cause severe symptoms, the optimal and unified approach and treatment have not been yet established. [7] The typical patient with VADA present with sudden severe occipital headache and posterior nuchal pain, followed by focal neurologic deficits attributable to brainstem or cerebellar ischemia $[8,9]$.

It is well known that a tear in arterial wall is capable of blood collection between the layers of the artery, known as intima, the inner layer, media, the middle muscular layer and adventitia, the outer layer. Mentioned event will lead to intramural hematoma formation. Healing process includes leukocytes and phagocytes infiltration, followed by epithelial cell coverage on the lesion and appearance of new smooth muscle cells to create a new intima layer. [10] Catheter angiography is the gold standard diagnostic test for VADAs, but due to its invasive nature and later complications, trends are toward non-invasive imaging modalities with potential to demonstrate small intramural hematoma, such as MRI and MRA. Intramural VA hematomas can be found as pearl-and-string and double lumen sign on MRA. Also, intramural hematoma which has a crescentic shape adjacent to the vessel lumen is suggestive of dissection on MRI. Fat suppression technique is important to differentiate small intramural hematomas from the surrounding soft tissues. [11] In addition, predicting role of Basi-parallel anatomic MRI scan in identifying surface contour of the intracranial vertebro-basilar artery for clinical course assessment of un-ruptured VAs has been investigated. [12,13,14] In our series, patients underwent diagnostic MRI and MRA during long-term follow-up. Complete re-canalization of a taperd occlusion requires a relatively long time. [11] Also, re-canalization was detected in patients with non-tapered occlusions or isolated string sign on angiography. [15] Although un-ruptured VADAs tend to have relatively benign prognosis, Naito et al observed SAH in 3 patients out of 21 with un-ruptured VADA, suggesting variable bleeding rate of these lesions. [16] Also in another study by Tsutsumi et al, 9 patients experienced $\mathrm{SAH}$ with previous un-ruptured VADAs at mean 6.7 days after initial complaints. [17] Mentioned studies suggest that patient's prognosis will improve if symptom-free interval lasts for more than 4 days. The results of our series advocate benign course of VADAs. Based on a study, about $3.5 \%$ of VADAs which cause ischemic focal neurologic symptoms resulted in SAH. [18] Lihara et al found follow-up angiography beneficial in patients with 
un-ruptured VADAs with concurrent aneurysmal formation [19] Patients with un-ruptured VADAs may require interventional occlusion, and consequent test. The blood flow from the anterior and posterior spinal arteries and the perforating arteries, as well as the cross-flow from the contra-lateral side and the relationship between the aneurysm and the PICA, must be monitored during the procedure. [20] Medical treatment for acute dissection of VA, include anticoagulation with intravenous heparin followed by oral warfarin regardless of symptoms, unless there are contraindications such as large infarct with mass effect and intracranial aneurysm. However, effectiveness of anticoagulant therapy in patients with ischemic symptoms is controversial, because they may promote dissection progression or increase aneurismal rupture risk. [21] Also, recurrent ischemia may be related to thrombotic occlusion of the stenotic lesion or perforating arteries from the VA due to progression of the dissection. In addition, Ro et al found that most of the patients with intracranial VA dissection encounter prodromal symptoms, emphasizes that early diagnosis of these lesions is necessary to prevent devastating SAH. [22] In our series, since no additional treatment was performed after $4^{\text {th }}$ month in follow-up period and all of the patients remained symptom free in $6^{\text {th }}$, $12^{\text {th }}$ and $24^{\text {th }}$ month, authors suggest minimum follow-up period between 4 and 6 months and no further work-up after 2 years.

\section{Conclusion}

Natural course of un-ruptured VADAs are not commonly aggressive. Early interventional endovascular interventions such as endovascular occlusion of the parent artery, endovascular trapping and intracranial stenting should be considered in patients with large or growing aneurysms. Base on the results of our study, authors suggest conservative management of un-ruptured VADAs and minimum follow-up between $4-6^{\text {th }}$ months an no further work-up after 2 years of initial symptoms.

\section{References}

[1] Kristensen, B., Malm, J., Carlberg, B., Stegmayr, B., Backman, C., Fagerlund, M., Olsson, T., "Epidemiology and etiology of ischemic stroke in young adults aged 18 to 44 years in northern Sweden,” Stroke, 28(9). 1702-9. 1997.

[2] Sato, T., Sasaki, T., Suzuki, K., Matsumoto, M., Kodama, N., Hiraiwa, K., "Histological study of the normal vertebral arteryEtiology of dissecting aneurysms," Neurologia medico-chirurgica, 44(12). 629-635. 2004.

[3] Nikouei, A., Seddighi, A., Seddighi, A.S., "The Results of Image Guided Surgery Using Neuronavigation in Resection of Cerebral Gliomas in Eloquent Cortical Areas," Archives of Physical Medicine and Rehabilitation, 97(10). e69-70. 2016.

[4] Haldeman, S., Kohlbeck, F.J., McGregor, M., "Stroke, cerebral artery dissection, and cervical spine manipulation therapy," Journal of neurology, 249(8). 1098-1104. 2002.
[5] Savitz, S.I., Caplan, L.R., Edlow, J.A., "Pitfalls in the diagnosis of cerebellar infarction," Academic emergency medicine, 14. 63-68. 2007.

[6] Pozzati, E., Padovani, R., Fabrizi, A., Sabattini, L., Gaist, G., "Benign arterial dissections of the posterior circulation," Journal of Neurosurgery, 75(1). 69-72. 1991.

[7] Yoshimoto, Y., Wakai, S., "Unruptured intracranial vertebral artery dissection. Clinical course and serial radiographic imagings,” Stroke, 28(2). 370-374. 1997.

[8] Kim, Y.K., Schulman, S., "Cervical artery dissection: pathology, epidemiology and management," Thrombosis research, 123(6). 810-21. 2009.

[9] Seddighi, A., Nikouei, A., Seddighi, A.S., Zali, A.R., Tabatabaei, S.M., Sheykhi, A.R., Yourdkhani, F., Naeimian, S., "Peripheral Nerve Injury: A Review Article,” International Clinical Neuroscience Journal, 3(1). 1-6. 2016.

[10] Mizutani, T., Kojima, H., Miki, Y., “Arterial dissections of penetrating cerebral arteries causing hypertension-induced cerebral hemorrhage,” Journal of Neurosurgery, 93(5). 859-862. 2009.

[11] Nakagawa, K., Touho, H., Morisako, T., Osaka, Y., Tatsuzawa, K., Nakae, H., Owada, K., Matsuda, K., Karasawa, J., "Long-term follow-up study of unruptured vertebral artery dissection: clinical outcomes and serial angiographic findings," Journal of Neurosurgery, 93(1). 19-25. 2000.

[12] Nagahata, M., Manabe, H., Hasegawa, S., Takemura, A., "Morphological change of unruptured vertebral artery dissection on serial MR examinations. Evaluation of the arterial outer contour by basi-parallel anatomical scanning (BPAS)-MRI," Interventional Neuroradiology, 12(suppl 1). 133-136. 2006.

[13] Takada, H., Hyogo, T., Kataoka, T., Hayase, K., Nakamura, H., "Diagnosis of vertebral artery dissection by basi-parallel anatomical scanning (BPAS) MRI,” Interventional Neuroradiology, 12(suppl 1). 129-132. 2006.

[14] Seddighi, A., Akbari, M.E., Seddighi, A.S., Pirayesh, E., Soleymani, M.M., Baqdashti, H.R., Nikouei, A., Zali, A., Tabatabaei, S.M., Naimian, S., Mellati, O., "Radioguided surgery using gamma detection probe technology for resection of cerebral glioma," Hellenic journal of nuclear medicine, 18(suppl 3). 68-75. 2014.

[15] Maillo, A., Diaz, P., Morales, F., "Dissecting aneurysm of the posterior cerebral artery: spontaneous resolution,” Neurosurgery, 29(2). 291-294. 1991.

[16] Naito, I., Iwai, T., Sasaki, T., "Management of intracranial vertebral artery dissections initially presenting without subarachnoid hemorrhage,” Neurosurgery. 51(4). 930-937. 2002.

[17] Tsutsumi, M., Kawano, T., Kawaguchi, T., Kaneko, Y., Ooigawa, H., "Dissecting aneurysm of the vertebral artery causing subarachnoid hemorrhage after non-hemorrhagic infarction," Neurologia medico-chirurgica, 40(12). 628-631. 2000.

[18] Yamaura, A., Isobe, K., Karasudani, H., Tanaka, M., Komiya, H., "Dissecting aneurysms of the posterior inferior cerebellar artery," Neurosurgery, 28(6). 894-898. 1991.

[19] Iihara, K., Sakai, N., Murao, K., Sakai, H., Higashi, T., Kogure, S., Takahashi, J.C., Nagata, I., "Dissecting aneurysms of the vertebral artery: a management strategy,” Journal of Neurosurgery, 97(2). 259-267. 2002.

[20] Shafigh, M., Fatouraee, N., Seddighi, A., "Determining the biomechanical properties of human intracranial blood vessels through biaxial tensile test and fitting them to a hyperelastic model,” Engineering Solid Mechanics, 1(2):43-56. 2013.

[21] Schievink, W.I., "Spontaneous dissection of the carotid and vertebral arteries," New England Journal of Medicine, 344(12). 898-906. 2001.

[22] Ro, A., Kageyama, N., Abe, N., Takatsu, A., Fukunaga, T., "Intracranial vertebral artery dissection resulting in fatal subarachnoid hemorrhage: clinical and histopathological investigations from a medicolegal perspective,” Journal of Neurosurgery, 110(5). 948954. 2009. 